\title{
Airway Complications and Outcome after Thyroidectomy in Ibadan: A 15 Year Review
}

\author{
Ayandipo $00^{1}$, Adigun $\mathrm{TA}^{2 *}$, Afuwape $00^{1}$, Afolabi $\mathrm{AO}^{1}$ and Daniel $\mathrm{A}^{3}$ \\ ${ }^{1}$ Department of Surgery, College of Medicine, University of Ibadan, Nigeria \\ ${ }^{2}$ Department of Anaesthesia, College of Medicine, University of Ibadan, Nigeria \\ ${ }^{3}$ Department of Otorhinolaryngology, College of Medicine, University of Ibadan, Nigeria
}

*Corresponding author: Adigun TA, Department of Anaesthesia, College of Medicine, University of Ibadan, Nigeria, Tel: 2349025058328; Email: tonitomi2005@yahoo.co.uk

Rec date: Jun 30, 2016; Acc date: Jul 18, 2016; Pub date: Jul 25, 2016

Copyright: (c) 2016 Ayandipo 00, et al. This is an open-access article distributed under the terms of the Creative Commons Attribution License, which permits unrestricted use, distribution, and reproduction in any medium, provided the original author and source are credited.

Citation: Ayandipo OO, Adigun TA, Afuwape OO, et al. Airway Complications and Outcome after Thyroidectomy in Ibadan: A 15 year review. Arch Med. 2016, 8:4

\begin{abstract}
Introduction: Thyroidectomy is one of the frequent major surgeries performed by the general surgery unit. There are challenges encountered with patent airway maintenance during preoperative, intraoperative and postoperative periods. The objective of this study was to review the airway complications and outcome after thyroidectomy in our centre.
\end{abstract}

Patients and Methods: This is a cross-sectional retrospective study of all the thyroidectomies performed from 2001-2015. The data collected included the demographic profile, postoperative airway complications and the outcome of management. Data was analyzed using SPSS version 16.

Results: Subjects included 507 patients. Majority were female $86 \%$, age ranged $19-83$ years and mean age of $38 \pm$ 16 years. Simple multinodular goiter was the commonest type. Postoperative airway complications were observed in $65(12.8 \%)$ patients. This constituted of haematoma in $4(0.8 \%)$ patients, laryngeal nerve injury in $15(3.0 \%)$, laryngeal oedema in $19(3.7 \%)$ and tracheomalacia in $27(5.3 \%)$ patients. Three patients died of respiratory complications consisting of two patients with tracheomalacia and one with primary retrosternal goiter.

Conclusion: Airway complications are not uncommon after thyroidectomy and tracheomalacia caused the highest morbidity and mortality.

Keywords: Goiter; Thyroidectomy; Postoperative airway complication

\section{Introduction}

One of the most common disorders of endocrine glands requiring surgical intervention is goiter. Thyroidectomy is the most common treatment for goiter presently in Nigeria [1]. Indications for thyroidectomy include multinodular goiter, thyroid cancer, Graves' disease and thyroiditis. However cosmesis is the most common indication generally [2].

The neglected goiter is still a problem in our environment and in some endemic areas; the enlarged glands reach enormous sizes before the patient gets to the surgeon [2]. The patients often present with symptoms related to tracheal or oesophageal compression or a sensation of pressure in the neck.

Airway management is a concern for both the anaesthetist and the surgeon in patients undergoing thyroidectomy. Challenges may be encountered during preoperative, intraoperative and postoperative periods. The enlarged thyroid is considered to be a risk factor for difficult laryngoscopy and failed intubation with associated respiratory complications [3]. A quoted incidence of difficult endotracheal intubation in thyroid surgery was $11.1 \%$ in a study by Amathiere et al. [4].

One of the indices of success in thyroid surgery is the frequency of complications. The most frequent postoperative complications following thyroidectomy are hypocalcemia and airway complications. These are life threatening and have a significant impact on quality of life [5]. Airway complications may result from postoperative haematoma, vocal cord paralysis, laryngeal oedema, and tracheomalacia.

In a study of 262 patients who underwent thyroidectomy in llorin teaching hospital in Nigeria, 20(7.6\%) patients had respiratory complications and the predictive factors for respiratory complication were giant goiter, tracheal narrowing or deviation, malignant goiters, duration of the goiter, preoperative status of the recurrent laryngeal nerves and retrosternal goiters. Sixty percent of their patients had 4 of these factors and $30 \%$ had 3 of these factors. The conclusion was that patients with at least 4 of these factors had a significant increased risk of developing postoperative respiratory complication [6]. 
The aim of this audit is to document the postoperative airway complications after thyroidectomy and identify factors that may contribute to complications and mortality in airway complication after thyroidectomy in our centre.

\section{Patients and Methods}

This is a retrospective review of all the patients who had thyroidectomy at University College Hospital, Ibadan, Nigeria over 15 years. Ethical approval was obtained for this study. Excluded cases were patients less than 16 years, emergency thyroidectomy and those done under regional anaesthesia.

Information was extracted from patients' clinical evaluation, supporting laboratory and imaging studies. The investigations included, full blood count, blood electrolyte, thyroid function test, soft tissue X-ray of the neck and thoracic inlet. Others were indirect laryngoscopy carried out by the otorhinolaryngologists and CT scan in suspected cases of retrosternal goiter.

Data collected included demographic profile, duration of goiter, presence of compressive symptoms (dysphonia, dyspnea, hoarseness, cough, or change in voice) and the thyroid position (cervical or retrosternal). Other data included number of attempt at intubation, use of bougie, use of fiberoptic laryngoscope/ bronchoscope and number of cases rescheduled, complications of surgery, postoperative tracheostomy and outcome of management such as complications and death.

There was a diverse range of anaesthetists of varying degrees of experience providing anaesthesia. If preoperative assessment was not predictive of airway difficulty, induction of anaesthesia was either with inhalational (halothane) or intravenous (propofol or sodium thiopentone) with or without muscle relaxants. Majority of the anaesthetists selected a conventional intravenous technique after test ventilation. Depolarizing muscle relaxants (suxamethonium) was used for endotracheal intubation. External laryngeal manipulation and bougie were the two commonly techniques in cases of difficult intubation. If preoperative assessment is predictive of difficult airway, fiberoptic guided nasotracheal intubation was used.

Postoperatively, patients were extubated awake in the theatre after reversal of residual neuromuscular blockade if there was no difficult intubation, suspected cases of recurrent laryngeal palsy or tracheomalacia. Otherwise patients were extubated 24-48 hours after surgery in the intensive care unit.

Data analyzed using SPSS version 16 . The mean, median and range were used for continuous variables whereas proportion and frequency were used for categorical variables.

\section{Results}

We studied 507 patients, 436 patients were females (86\%) and 71 were males (14\%) with a female to male ratio of $6: 1$. The ages ranged from 19-83 years with a mean age of 38 years and standard deviation of 16 . Patients from the rural areas were $48 \%$ and $52 \%$ from the urban area.
Preoperative findings were multinodular goiter $73 \%$, isolated thyroid nodule $3.5 \%$, neoplastic goiter $9.1 \%$ and Grave's disease in $12.2 \%$ and thyroiditis $2.2 \%$. Retrosternal extension goiter was in 43 patients (8.5\%) and the rest are cervical. Table 1 showed the patients' preoperative finding.

Table 1 Patients' preoperative finding.

\begin{tabular}{|l|l|l|}
\hline & $\mathbf{n}$ & $\%$ \\
\hline Multinodular goitre & 370 & 73 \\
\hline Isolated thyroid nodule & 18 & 3.5 \\
\hline Neoplastic goitre & 46 & 9.1 \\
\hline Graves disease & 62 & 12.2 \\
\hline Thyroiditis & 11 & 2.2 \\
\hline
\end{tabular}

All patients underwent at least 1 of the following preoperative imaging studies; chest radiography, CT scan or MRI.

Tracheal deviation was seen in 238 (47\%), tracheal compression in $192(37.5 \%)$ and retrosternal extension in $43(8.5 \%)$ patients.

Indirect laryngoscopy was normal in 495 patients. The left vocal cord was relatively immobile in 14 cases and on the right in 11 patients. The patients with vocal cords palsy had malignant goiter.

Endotracheal intubation was successful within the first three attempts in 406 patients, 67 patients were intubated after the third attempt, and 9 patients were rescheduled due to inability to intubate at all.

Thirteen patients had awake fiberoptic intubation done and the bougie was used in 47 patients for successful intubation.

Intraoperative assessment revealed adherence of the goiter to the tracheal in 9 patients, significant tracheomalacia in 27 patients and floating tracheal in 18 patients. Recurrent laryngeal nerves were identified in $472(93 \%)$ patients and not in $35(7 \%)$ patients.

Postoperatively, 455 patients were extubated in the theatre without any respiratory distress and transferred to the recovery room, 19 patients were re-intubated in theatre due to respiratory distress and transferred to the intensive care unit and tracheal extubation was delayed in 33 patients until after 24 hours postoperatively in the intensive care unit.

Sixty five patients (12.8\%) had postoperative airway complications. Four patients $(0.8 \%)$ had postoperative neck haematoma that was detected in recovery room and required immediate reoperation and evacuation of clot.

15 patients (3.0\%) had transient laryngeal nerve injury and 19 patients $(3.7 \%)$ had laryngeal oedema that could not be extubated within 6 hours postoperatively.

27 patients (5.3\%) had tracheomalacia that required elective tracheostomy in 10 patients $(2.0 \%)$ and 17 patients $(3.3 \%)$ had 
tracheotomy within 24-96 hours postoperatively. Table 2 showed the postoperative airway complications.

Table 2 Postoperative airway complications.

\begin{tabular}{|l|l|l|}
\hline & N & $\%$ \\
\hline Haematoma & 4 & 0.8 \\
\hline Recurrent laryngeal Nerve palsy & 15 & 3 \\
\hline Laryngeal Oedema & 19 & 3.7 \\
\hline Tracheomalacia & 27 & 5.3 \\
\hline
\end{tabular}

Three postoperative mortalities were found in this series, 2 patients died due to tracheomalacia and one due to primary retrosternal goiter.

\section{Discussion}

In this study the mean age and female male ratio was $38 \pm$ 16 and 6:1 respectively similar to a study in Ethopia [7]. However the sex ratio was 8.6:1 in a study by Soyannwo et al. from the same institution [2]. The age range was 19-83 years compared to $19-89$ years found in the earlier study7.

Multinodular goiter (73\%) was the commonest type in this study similar to many other studies $[2,7]$. This by nature tends to cause progressive growth and expansion to surrounding structures leading to compressive symptoms and tracheal deviation.

The incidence of airway complications in this study was $12.8 \%$ more than $7.6 \%$ obtained by Rahman 6 and less than $31 \%$ reported by Abdel Rahim et al. [8] although their series involved predominantly large goiters. Causes of airway problem in this study included haematoma, recurrent laryngeal nerve injury, laryngeal oedema and tracheomalacia.

Post thyroidectomy haematoma was found in 4 patients $(0.8 \%)$ in this study. This is lower contrary to previously reported incidence of $1.3 \%$ by Shaha et al. [9], $2.5 \%$ by Agrawal et al. [10] and higher than $0.4 \%$ reported by Lacoste et al. [11]. Haematoma occurred within the first 6-8 hours after surgery and manifested as increased blood in the drain, neck pain, and respiratory distress. It is usually an emergency requiring evacuation of the haematoma under general anaesthesia. Intubation during re-exploration may be difficult due to oedema of the epiglottis, pharyngeal walls and vocal cords. Bleeding may sometimes be large enough to require fluid and blood resuscitation however; only one patient had blood transfusion amongst the four that had haematoma in this series.

Unilateral recurrent laryngeal nerve palsy (RLNP) was found in $15(3 \%)$ of patients and no patient developed bilateral RLNP postoperatively in this study. Rahman recorded $10 \%$ of patients with respiratory complication were attributed to RLNP6 while Jamski et al. reported ranges of 0.5 to $5 \%$ depending on the type of thyroid surgery performed [12]. Low rate of RLNP could be explained by the fact that routine visual identification of the nerve during surgery is practiced in our centre. Numerous techniques have been used to identify the nerves and prevent its damage [13]. Kartz et al. used an operative microscope to identify and stimulate the RLN with visualization of the glottis with a laryngoscope during surgery [14]. The use of intramuscular electrode to detect laryngeal muscle electromyography (EMG) activity in response to RLN stimulation was also documented by Davis et al. [15]. However, Freschi et al. reported that the only sure method of preventing RLN damage is visual identification [16].

In addition, $3.7 \%$ of patients had laryngeal oedema requiring immediate re-intubation after extubation. This is usually indicative of multiple attempts at intubation, trauma to vocal cord during intubation and excessive dissection at surgery. Martis et al. showed that frequent attempts at intubation may result in fatal laryngeal oedema with incidence of about $1 \%$ [17]. Higher incidence in this study may be due to the multiple attempts at endotracheal intubation observed. Majority of the patients had intubation achieved within the first three attempts while 67 patients were successfully intubated after third attempt. Few patients had to have their surgery rescheduled due to difficult/ failed intubation.

The most common airway complication found in this study was tracheomalacia in 27 patients (5.3\%). This was lower compared with $16 \%$ reported by Abdel Rahim et al. [8] although they studied mainly patients with long standing giant goiters. Majority of the patients with tracheomalacia in our series also had huge and long standing goiter with pressure symptoms and postoperative elective tracheostomy was performed in 10 patients and 17 patients had tracheostomy after 24-96 hours postoperatively. Some authors do advocate a planned tracheostomy especially in the presence of identifiable risk factors of respiratory complication [18]. Previous studies have shown that tracheomalacia was the most common indication for tracheostomy [8]. However, Rahman in Nigeria reported zero incidence of tracheomalacia in his study [6].

This study showed that three patients died due to airway compromise, two patients had tracheomalacia and the last had primary retrosternal goiter that was not detected at surgery. Sano et al. study reported two deaths out of 82 patients [19] while Rahman's study showed no mortality [6].

In conclusion, airway complication is a common postoperative complication encountered after thyroidectomy. Tracheomalacia is the most common airway complication in our series and predisposing factor was prolonged huge thyroid gland. Anticipation of this problem and prompt management will give a better outcome in patients undergoing thyroidectomy.

\section{References}

1. Famuyiwa OO (1990) Problem and challenges in the practice of endocrinology in developing countries; Thyroid disease. Nigeria medical practitioner 20: 87-91.

2. Soyannwo OA, Ajao OG, Agbejule OA, Amanor- Boadu SD (1995) Anaesthesia and surgical aspects of thyroid swellings. The Ibadan experience. E Afri Med J 72: 675-677. 
3. Cobley M, Vanghan RS (1992) Recognition and management of difficulty airway problem. BJA 68: 90-97.

4. Amathiere R, Small N, Catineu J, Poloijadoff MP, Samil K, et al. (2006) Difficult intubation in thyroid surgery myth or reality? Anaesth Analg 103: 965-68.

5. Slaweta N, Gluszek S (2012) The effect of complicated thyroid surgical procedures on personal and professional life of patients. Pol Przegyl chir 54: 437-44.

6. Rahman GA (2009) Possible risk factors for respiratory complications after thyroidectomy; an observational study. Ear Nose Throat J 88: 890-892.

7. Mengistu M (1993) The pattern of thyroid disease in adult Ethiopian and experience in management. Eth MED J 31: 25-36.

8. Rahim AA, Ahmed ME, Hassan MA (1999) Respiratory complications after thyroidectomy and the need for tracheostomy in patients with a large goiter. Br J Surg 86: 88-90.

9. Shaha AR, Jaffe BM (1994) Practical management of post thyroidectomy heamatoma . J Surg Oncol 57: 235-238.

10. Agrawal A, Mishra S (1997) Post-thyroidectomy haemorrhage An analysis of critical factors in successful management. J Indian Med Assoc 95: 418-419.

11. Lacoste L, Gineste D, Karayan J (1993) Airway complications in thyroid surgery. Ann Otol Rhinol Laryngol 102: 441-446.
12. Jamski J, Jamska A, Graca M, Barczynski M, Wlodyka J (2004) Recurrent laryngeal nerve injury following thyroid surgery Przegl Lek 61: 13-16.

13. Chiang FY, Lee KW, Huang YF, Wang LF, Kuo WR (2004) Risk of vocal palsy after thyroidectomy with identification of the recurrent laryngeal nerve. Kaohsiung J Med Sci 20: 431-436.

14. Kartz RC (1972) The identification and protection of the laryngeal motor nerve during thyroid and laryngeal surgery. A new microsurgical technique. Laryngoscope 83: 59.

15. Davis WE, Rea JL, Tempter B (1979) Recurrent laryngeal nerve localization using a micro laryngeal electrode. Otolaryngeal Head Neck Surg 87: 330.

16. Freschi G, Mast C, Pichi, Graziani M (1994) Anatomic and surgical consideration regarding the recurrent laryngeal nerve in thyroidectomy. Mnrva Chir 49: 943-947.

17. Martis C, Athanasside SS (1971) Post thyroidectomy laryngeal oedema ; a survey of fifty four cases. Am J Surg 122: 58.

18. Bennette AMD, Hashmi SM, Premachandra DJ, Wright MM (2004) The myth of tracheomalacia and difficult intubation in cases of retrosternal goiter. Layngol Otol 118: 778-780.

19. Sano D, Ouba R, Wandaogo A, Sanon A, Sondre BR (1995) Problem after throid gland surgery in Bakina Faso; 83 cases. Med Trop Mars 55: 51-54. 